\title{
Real Time Fake Currency Note Detection using Deep Learning
}

\author{
M. Laavanya, V. Vijayaraghavan
}

\begin{abstract}
Great technological advancement in printing and scanning industry made counterfeiting problem to grow more vigorously. As a result, counterfeit currency affects the economy and reduces the value of original money. Thus it is most needed to detect the fake currency. Most of the former methods are based on hardware and image processing techniques. Finding counterfeit currencies with these methods is less efficient and time consuming. To overcome the above problem, we have proposed the detection of counterfeit currency using a deep convolution neural network. Our work identifies the fake currency by examining the currency images. The transfer learned convolutional neural network is trained with two thousand, five hundred, two hundred and fifty Indian currency note data sets to learn the feature map of the currencies. Once the feature map is learnt the network is ready for identifying the fake currency in real time. The proposed approach efficiently identifies the forgery currencies of 2000, 500, 200, and 50 with less time consumption.
\end{abstract}

Keywords: Convolutional neural network, Currency detection, Deep learning, Feature extraction, Image processing.

\section{INTRODUCTION}

Counterfeiting refers to an illegal copy of the currency of origin. Therefore, counterfeit currency is not approved by the government. RBI is the only body responsible for printing banknotes in India. Every year, the RBI has to deal with the problem of counterfeit banknotes once filtered and placed on the market.

Currently with the development of better image processing methods, new methods for identification of currency is designed by analysing specific safety information present in the currency. The safety features are watermarks, hidden images, security threads, and optically variable inks. Therefore, to determine the currency using image processing, extract the specific statistics from the currency image and select the correct recognition approach. The main methods for currency recognition is by characteristic geometric size [1] and by characteristic texture [2]. The general steps followed by image processing approach is to acquire image, to detect edge, to convert image to gray scale, feature extraction, image segmentation and decision making [3-4]. The drawback of these approaches are detection efficiency is less since feature extraction is a challenging task. To overcome this problem now the trend is towards deep learning, since it is a multilayer neural network. The deep neural network is effective for different application in real time.

Revised Manuscript Received on December 15, 2019

M. Laavanya, Department of Electronics and Communication Engineering, Vignan's Foundation for Science, Technology and Research, Vadlamudi, Guntur, AP. Email: laavanvijay@gmail.com

V. Vijayaraghavan, Department of Electronics and Communication Engineering, Vignan's Foundation for Science, Technology and Research, Vadlamudi, Guntur, AP. Email; vijayaraghavan123@gmail.com
In general, to form a deep neural network, we need a large set of image data for the activity to be done. But thanks to transfer learning technique, we only need a small amount of data sets. What we do is take a model already trained in a large data set and use our weights to reconstruct the small data set we have. In this way, a large data set is not necessary and the model is also designed correctly. Hence in this paper transferred learned Alex net is used by fine tuning the last layer of this model to get the desired accuracy.

\section{RELATED WORKS}

Concerns have been raised in recent years about the currency recognition system due to increase in counterfeit currency circulation. Hence the objective of any currency recognition system is to find the fake currency. A thriving approach to identifying paper money depends on a series of steps, first to capture image, then conversion to grayscale, detection of edges, segmentation, feature extraction and image comparison. In this paper, we viewed at diverse types of literature that describe different methods of identifying counterfeit currencies. The document also provides an overview of methods to identify counterfeit Indian currency for fraud detection. Currency detection will work efficiently by applying some effective pre-processing and recovery methods [5]. One of the most effective methods of counteracting counterfeiting can be to use easily accessible and effective software to detect counterfeit currency [6]. A method for determining the currency in real time for classifying the currency is proposed. The currency of image acquisition is based on currency discovery, when the size of various denominations is obtained, and the features are extracted, it gives the information to Kohonen's training network. This scheme is good with high speed and recognition accuracy [7]. In [8] support vector machine based currency recognition is presented. It segments our currency into various regions with our own core, linear weighing through various forms of basic training matrices and is studied using semi-defined programming, are used to obtain ideal weights. Use of RGB and HSV color space model in the image processing, helps in currency detection with new 500 and 2000 notes. It can be detected faster by making samples of these banknotes. This system used for automatic currency recognition based on image processing [9]. The currency image is represented in the space of differences, which is a vector space constructed by comparing the image with a series of prototypes. Each measurement measures the difference between the image in question and the prototype. To distinguish between two images, the local key points of each image are identified and described. Based on currency characteristics, it is possible to effectively identify the relevant key points between two images.

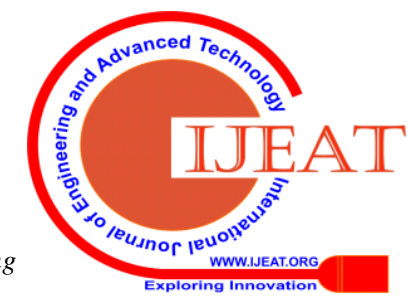


Due to the limited number of real-life counterfeit currencies, SVM is used to detect counterfeit currencies, so only authentic currencies are required for authentication and to train classifier [10].

In recent years counterfeiting using neural network is good at detection. In [11] it was found that the functions were passed through direct feed forward neural network (FNN) and is therefore used for currency recognition as well as verification. To detect, it uses the color information mined from currency, but it leads to high risk of error and also makes the experimental results to be less accurate. An approach with back propagation network and principal component analysis when used for feature extraction, the approach is successful with an additional investigation [12]. In convolutional based neural network when the number of layers are more then complexity is also more [13]. But convolution neural network is robust and detection speed is faster [14]. In [15], the problem of stochastic gradient slope (SGD) is been explored and points out due to SGD tilt, the previous set of random parameters drops sharply then it may affect the overall performance of the detection process. Hence in our presented method, we have used convolutional neural network with adam optimization to detect fake currency note.

\section{METHODOLOGY}

In this proposed system, our relevance is to focus on detection of fake currencies which is spread in Indian market. In our work fake currency is identified by extracting the security thread feature in the currency note. For detecting the fake currency, the most popular method in deep neural network called transfer learning using Alex net is adopted.

Alex net consists of convolutions, max pooling, dropout, ReLU activations and fully-connected layers. The layers are as displayed in below figure 1. In transfer learning the last three layers are fine tuned to accommodate the requirement of our proposed work. The ' $\mathrm{fc} 8$ ' layer is tuned with a weight learning factor and bias learning factor for accurately extracting the latent feature from the image. The weight gives the impact of the input on the network and bias is used to adjust the output with weighted sum of inputs to the neuron. The accuracy of learning feature grows with the weight and bias learning factor.

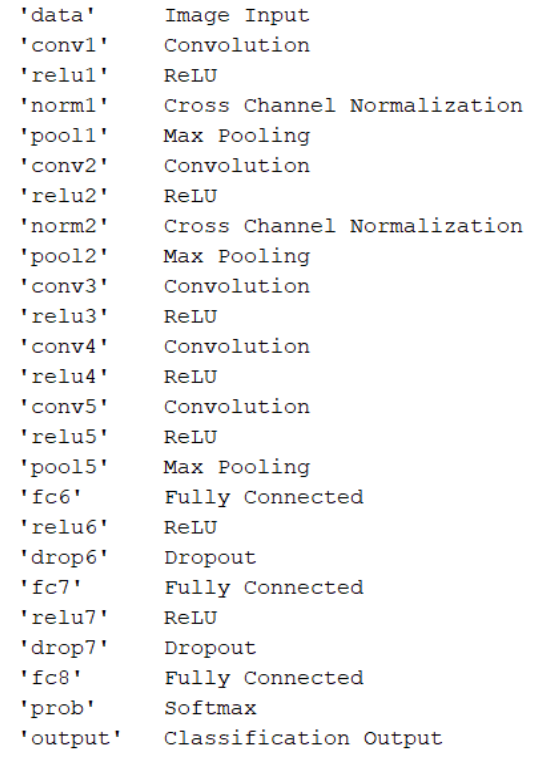

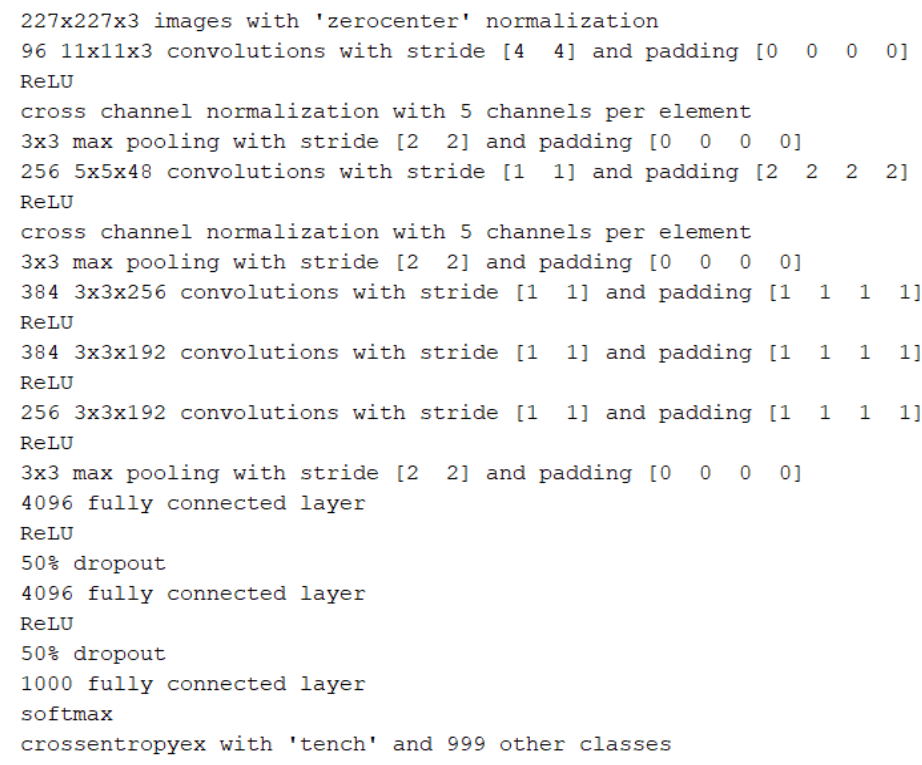

Figure 1. Layers of Alex net
The input image is usually two-dimensional, so the object map is also two-dimensional. The convolution layer applies a specified number of filters to the input image. The mechanism is as follows. The filter is an array of numbers the same size as the input image. This filter moves to each pixel value and multiplies by each filter number by the corresponding pixel value in which it is positioned and calculates multiplication by element. For each pixel position, each multiplication is added to make it an element of the output matrix called a feature map, as shown in the following figure 2 .

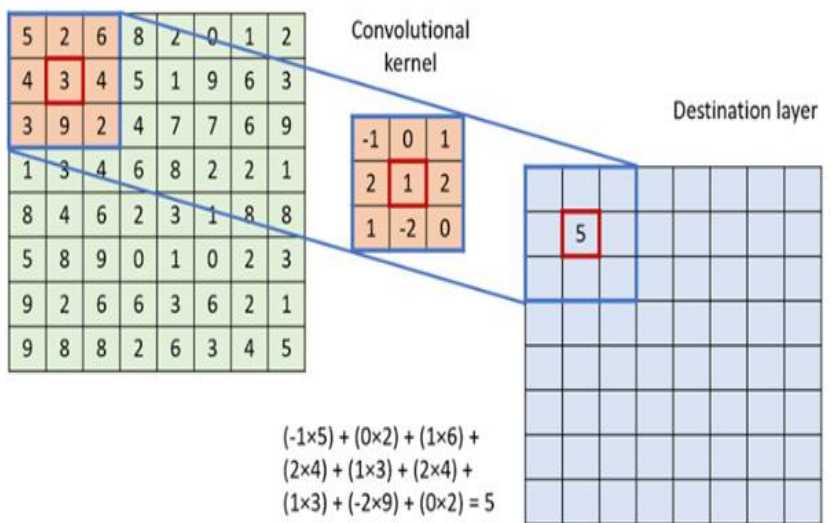

Figure 2. Convolution operation

Another parameter to consider is the stride. This is the amount of pixels that the strainer matrix moves each time it is moved to a different position on the input image. As a general rule, the passage is 1 , which allows us to leave

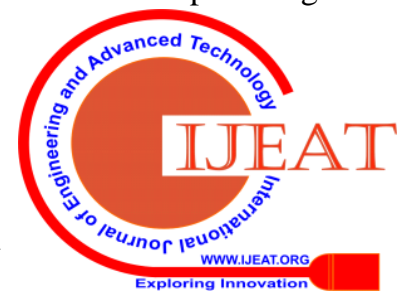


all the decreasing spatial samples for POOL levels.

An element of the smart activation function is applied after each convolution layer. Using the activation function, network linearity is interrupted to detect more complex linear regression functions. The function for activating a rectified linear unit is defined as $\max (0, \mathrm{x})$. The spatial contact of the output data obtained from the single feature map and the total values of the secondary matrix value help to describe the value of the physical domain. Select maximum grouping, which simply shows maximum activation as seen in the region. The maximum coupling operation for a $2 \times 2$ matrix is shown in the following figure 3.
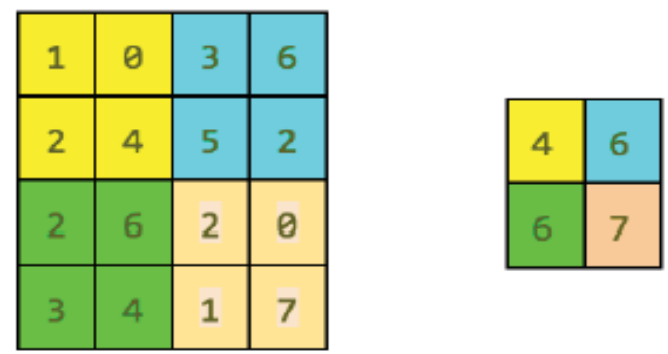

Figure 3. Max pooling operation

After several layers of convolutional and maximum pooling, high-level neural network reasoning is done through fully connected layers. The term "fully coupled" means that each neuron from the previous level is associated to each neuron

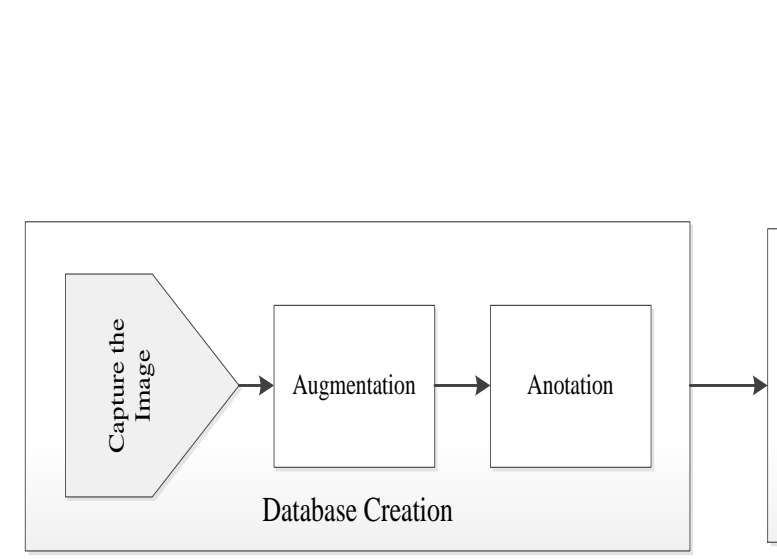

Figure 4. Work flow of proposed scheme

\section{EXPERIMENTAL RESULTS}

The proposed method uses transfer learned Alex network with adam optimization. The developed network is tested with a database of 100 images per currency, by taking fifty images as such as captured image and another 50 images are augmented images. The database image of Indian currency 50, 200, 500 and 2000 real notes are shown in figures. The detection of real currency rate is shown in table 1 . The to the next level. High level features can be extracted from the convolutional and pooling layers. The determination of a fully connected layer is to use these characteristics to sort the original image into different types based on a training dataset.

\section{TRAINING THE NETWORK}

To train the network, the images of currency note data base is generated. For each note 100 images are generated using augmentation. The augmentation process like resizing and rotating is carried out to increase the data base count. After augmentation annotation of all currency images is done and then the images are stored in a separate folder with labelling. Now the network and images are ready to be trained. Once the training process is over the network learns the features of real currency note of 2000,500,200 and 50 .

The system used for training is Intel Core i3 64-bit processor @ 2GHz with 4-GB RAM with Windows 10 and Matlab 2018a. The proposed scheme objective is to learn more features using fully connected layer. For this optimization hyper parameter is used to grow up the learning feature. The method is tested in real time using webcam, as soon as the image is captured the network starts learning the feature of the input currency note and compares with the learned features and gives the result as "Real Note" (or) "Fake Note". The stream of the process is displayed in figure 4.

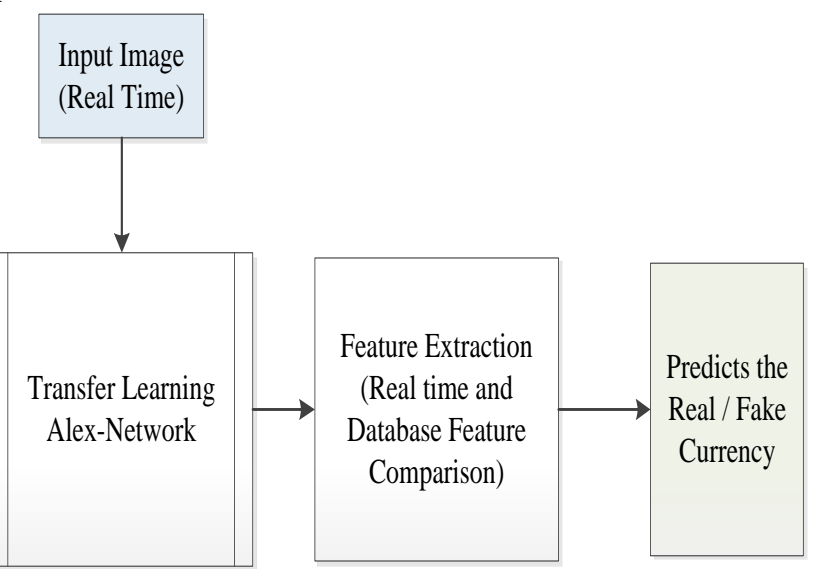

scheme has been verified with the use of authentic Indian currencies and counterfeit currencies. The average accuracy for the real currency and counterfeiting was $81.5 \%$ and $75 \%$, respectively. The total processing elapsed time of the model is 3 seconds. The proposed system is better by an approximate accuracy of $26 \%$ than VGG network [16]. The performance is better but still it can be improved by increasing the database count. 

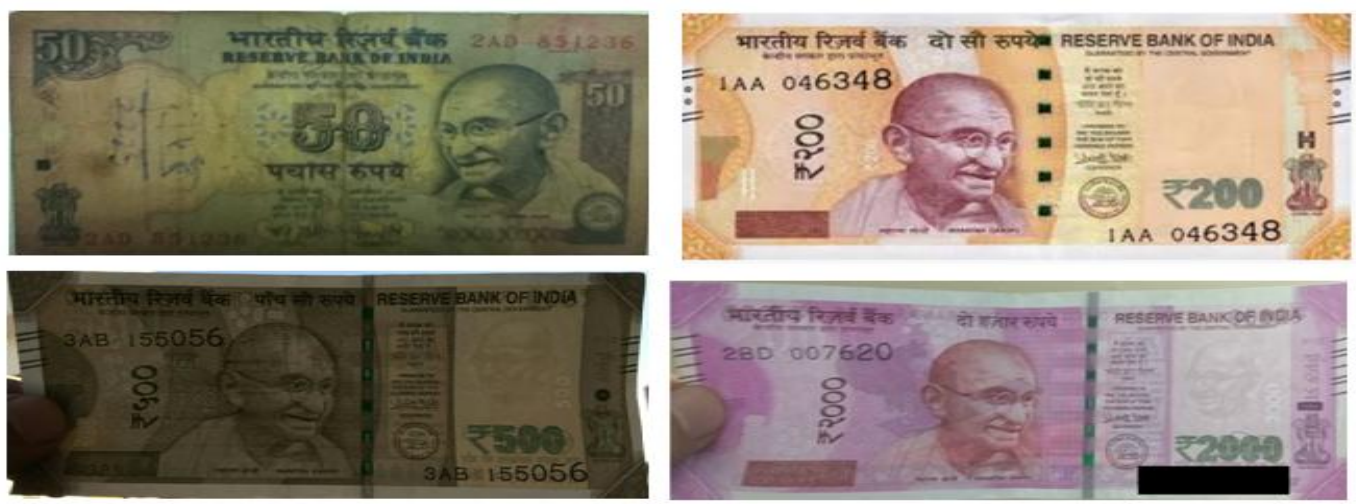

Figure 5. Database image of currency a) 50-rupee b)200-rupee c) 500-rupee d) 2000-rupee

Table 1. Experimental Results of the transfer learning Alex Network.

\begin{tabular}{|c|c|c|}
\hline Currency & Real Note & Fake Note \\
\hline 50 & $81 \%$ & $79 \%$ \\
\hline 200 & $82 \%$ & $78 \%$ \\
\hline 500 & $82 \%$ & $79 \%$ \\
\hline 2000 & $87 \%$ & $82 \%$ \\
\hline
\end{tabular}

\section{CONCLUSION}

In this paper transfer learned Alex net as our model for performing the fake currency detection. The detection accuracy is most accurate since the currency characteristics features are learned through layer by layer. Here we have considered the whole currency image, but in future we will try to include all the security features of currency by employing suitable structural design and with suitable training data. Further, noise may be present in the captured image which has to be considered as a pre-processing step $[17,18,19]$ in currency detection process. The recognition and fake currency detection can also be extended by considering the patterns of currency surface as features for improving the detection accuracy.

\section{REFERENCE}

1. D-F Wang, and S-W Lian, "Automatic selling tickets machine on railway station and cash recognition technology", Computer System Applications, Vol. 7, pp. 12-14, 1999.

2. Z-X Ymg, Z-B Qian and J-G Li. "Currency recognition using mathematical morphology and neural networks", Journal of Shanghai Jiaotong University, Vol. 33, No. 9, pp. 1142-1145, 1999.

3. R Mirza, and V Nanda, "Design and implementation of Indian paper currency authentication system based on feature extraction by edge based segmentation using Sobel operator", International Journal of Engineering Research and Development, Vol. 3, No. 2, pp. 41-46, 2012.

4. J Guo, Y Zhao, and A Cai, "A reliable method for paper currency recognition based on LBP", IEEE International Conference on Network Infrastructure and Digital Content, pp. 359-363, 2010.

5. PJ Grace, and A Sheema, "A survey on fake Indian paper currency identification system", International Journal of Advanced Research in Computer Science and Software Engineering, Vol. 6, No. 7, July 2016.

6. ST Gouri, PK Akshay, M Sneha, and S Bharat, "Detection of fake Indian currency", International Journal of Advance Research, Ideas and Innovations in Technology, Vol. 4, No. 2, pp. 170-176, 2018.

7. EH Zhang, B Jiang, JH Duan, and ZZ Bian, "Research on paper currency recognition by neural networks. In: International conference on machine learning and cybernetics, Vol. 4, pp. 2193-2197, 2003

8. CY Yeh, WP Su, and SJ Lee, "Employing multiple-kernel support vector machines for counterfeit banknote recognition", Applied Soft Computing (Elsevier), Vol. 11, No. 1, pp. 1439-1447, 2011.
9. MA Kumar, B Bhuvaneswari, and D Dhanasekaran, "Detection and recognition of counterfeit currency notes", International Journal of Pure and Applied Mathematics, Vol. 119, No. 17, pp. 1535-1540, 2018.

10. M Patil, J Adhikari, R Babu, "Fake currency detection using image processing", International Journal on Future Revolution in Computer Science \& Communication Engineering, Vol. 4, No. 4, pp. 865-868, 2018.

11. J Chambers, "Digital currency forensics", Masters dissertation, Auckland University of Technology, 2013.

12. Y Ren, "Banknote recognition in real time using ANN", Masters dissertation, Auckland University of Technology, 2017.

13. J Schmidhuber, "Deep learning in neural networks: An overview", Neural Networks (Elsevier), Vol. 61, pp. 85-117, 2015.

14. Q Zhang, WQ Yan, and M Kankanhalli, "Overview of currency recognition using deep learning" Journal of Banking and Financial Technology

15. I Sutskever, J Martens, G Dahl, and G Hinton, "On the importance of initialization and momentum in deep learning", 30th International Conference on Machine Learning (PMLR), Vol. 28, No. 3, pp. 11391147,2013

16. KG Navya, GS Pooja, BN Ram, VY Radha, and P Rajarajeswari, "Recognition of Fake Currency Note using Convolutional Neural Networks", International Journal of Innovative Technology and Exploring Engineering, Vol. 8, No. 5, pp. 58-63, 2019.

17. M Laavanya, and V Vijayaraghavan, "A sub-band adaptive visushrink in wavelet domain for image denoising", International Journal of Recent Technology and Engineering, Vol. 7, No. 5S4, pp. 289-291, 2019.

18. V Vijayaraghavan, and M Karthikeyan, "Denoising of images using principal component analysis and undecimated dual tree complex wavelet transform", International Journal of Biomedical Engineering and Technology (Inderscience), Vol. 26, No. 3-4, pp. 304-315, 2018.

19. V Vijayaraghavan, M Laavanya, and M Karthikeyan, "Real oriented 2-D dual tree wavelet transform with non-local means filter for image denoising", Journal of Electrical Engineering, Vol. 17, No.2, pp. 106$111,2017$. 